\title{
Grey Wolf Optimization and Crow Search Algorithm for Resource Allocation Scheme in Cloud Computing
}

\author{
Jiarui Wang \\ College of Information Science and Engineering \\ Northeastern University, \\ Boston, Massachusetts, United States \\ jiaruiwang698@gmail.com
}

\begin{abstract}
The computing resources are supplied by cloud computing on basis of cloud user requirements demand. Using virtualization and distributed computing, the resource allocation model is constructed to highlight the cloud services scalability. Nevertheless, a complex problem is created by the user, to manage the demand in the on-demand resource allocation model. Hence, a novel optimization approach is developed called Grey Wolf Optimization and Crow Search Algorithm (GWO-CSA) to resolve the problem in the resource allocation model. On the basis of the availability of the resources, the tasks are executed with the aid of the virtualization concept, minimize the response time. In a distributed manner, to the virtual machine, the tasks are allocated, to balance workload in the cloud. The proposed optimization method is exploited to attain effectual resource allocation. Finally, the developed method performance showed that it attains the utmost resource consumption, utmost memory consumption, and utmost CPU utilization, least skewness.
\end{abstract}

Keywords: Cloud Computing, Tasks, User Requirements, Virtual Machines, Work Load

\begin{tabular}{ll} 
Nomenclature & \\
\hline Abbreviations & Descriptions \\
\hline SOA & Service Oriented Architecture \\
VM & Virtual Machine \\
MKP & Multidimensional Knapsack Problem \\
ACO & Ant Colony Optimization \\
EC & Evolutionary Computation \\
PM & Physical Machine \\
DFLS & Dynamic Fuzzy Learning Strategy \\
GA & Genetic Algorithm \\
VMP & Virtual Machine Placement \\
PSO & Particle Swarm Optimization \\
FCFEDARA & Feedback-based Combinatorial Fair Economical Double Auction Resource Allocation Model \\
GWO & Grey Wolf Optimization \\
IWD & Intelligent Water Drop \\
BQP & Binary Quadratic Programming \\
CSA & Crow Search Algorithm \\
HDCSN & Hierarchical Distributed Cloud Service Network Model \\
\hline
\end{tabular}

\section{Introduction}

Generally, cloud computing is considered as the technical solution for various issues in several fields namely healthcare, business, and education fields. Cloud computing presents a group of computing, storage other resources which are used over the network. In the cloud, the application present is the inheriting element of the day-to-day human beings' lives. In the form of cloud services, the cloud model increases the functionalities that can be collected to create the applications of the cloud. In general, the cloud services are modeled in traditionalism with the SOA principles [1].

To provide on-demand computing services, cloud computing includes technology, and also it has come out as the information technology horizon [11]. Because of the cloud computing capability to access the cloud from everywhere, it received worldwide notice. In the cloud data center, one of the important 
technologies exploited is the VM beyond numerous technological elements. For environmental isolation server consolidation, the VM technology is an obliging hopeful. There is a sudden rise in the VM deployment because of the pointed increase in the services demands; it has been seen in the cloud data centers. Huge amounts of hosts are needed to convene the requirements of VM allocation that outcomes in a raise in energy utilization the complete execution cost. There is a requirement for effectual VM allocation policies that minimize energy utilization reduces obligatory VM migration and active servers. By idle host shutting down, the energy utilization is reduced, from minimum loaded hosts the VM can be migrated to hosts possessing average load [2].

The energy utilization can be reduced by using the optimal VM allocation policies, by positioning the $\mathrm{VM}$ on the heterogeneous and homogeneous resources effectively.

In cloud computing, resource allocation is considered a significant matter. Users submit their requirements on the cloud platform that include the time constraints like execution time, arrival time deadline, and multiple resource requirements [12]. Hence, a sensible resource allocation model should be modeled. Numerous resource allocation issues can be transferred into an MKP or its variants. In cloud computing, the auction mechanisms are introduced by the resource providers to permit the idle resources to be sold for higher profit at a dynamic price [3].

The dynamic scheduling model comprises two diverse factors like system state estimation and decision making in the static model the cost estimation. Generally, the EC techniques, such as GA are extensively exploited to improve resource utilization and energy utilization. Nevertheless, for VMP, the GA technique improved with the fuzzy multi-objective system is developed. To present the optimal solution, metaheuristic techniques like ACO, GA, and PSO are developed. The optimization algorithm called GWO is developed that is the motivation of grey wolves. In addition, the performance is calculated based on the matching level which subsists among the system model and meta-heuristic technique [4].

The main contribution of this work is to present a resource allocation model on the basis of the proposed GWO-CSA model, which is the combination of GWO and CSA model, this method is exploited to improve the resource allocation scheme performances. The task is allocated by the proposed method in an efficient manner to VM by taking into consideration of the minimum fitness value.

\section{Literature Review}

In 2020, Ritu Singhal and Archana Singhal [1], developed an FCFEDARA, which examines the authenticity of providers on the basis of the accessible prices for the needed resources besides to the feedback stated by the consumers. The adopted model presents a general platform for the consumers to access the resources from diverse providers at the best prices. The developed technique pays attention to the trustworthiness of the presenters, and the provider's priority to those presents that possess the better customer feedback.

In 2020, Jixian Zhang et al [2], addressed the issue of online time-deviating the multidimensional resource pricing as well as allocation in clouds. In particular, for the time-varying multi-dimensional resource allocation issue, a new integer programming model was proposed. For resource allocation, an ingenious online sale model in a gung ho environment was proposed. A waiting period scheme and dominant-resource-based scheme were developed to enhance the social welfare and resource utilization for the resource allocation technique.

In 2020, Kalka Dubey and S.C. Sharma [3], developed the IWD technique and VM allocation technique were proposed that optimizes task execution in a protected cloud environment. In the CloudSim simulation toolkit, the developed model was examined in cloud computing, and to verify the authority of the technique, an analysis was carried out with other renowned VM allocation policies.

In 2020, Jing Li [4], developed the HDCSN with the aid of the 5G system. This model comprises three kinds as Distributed Micro cloud, Access Cloud, and Core cloud that convenes the fundamental needs of the network system model. Based on PSO, a multi-node system allocation algorithm and resource graph scheduling were modeled.

In 2020, Junyan $\mathrm{Hu}$ et al [5], developed a model to exhibit collaborative communications among consumers and ascertain the number of service programs bought from each supplier to charge each cloud consumer the least price. Based on the multi-consumer resource procurement, a coalition was established. Then it shows that there subsists a different optimal solution in the coalition game.

In 2020, Christina Terese Joseph and Prof. K. Chandrasekaran [6], modeled microservice application modules that was deployed in the cloud infrastructure and it were named as a BQP issue. The adverse communication latencies impact was minimized on response time, communication pattern among microservice modules was designed. In Kubernetes, the developed allocation policies were developed. 


\section{Cloud Model}

The cloud users permit the retrieve, store, and process of the information concurrently in the cloud computing model [7], the cloud handles the larger amount of data per sec. The PM and VM are the cloud resources that execute the task on basis of the user request. To present the cloud services, VM migration objectives dynamically act in response to the request of the user. To carry out the appropriate task in the cloud, the resource allocation technology allocates the resources.

The cloud operation and degradation in performances, which creates the cloud model in an ineffectual manner, therefore it is needed to model the resource allocation scheme highly cautious manner. In addition, every task possesses its individual runtime and deadline. The task is allocated by the resource allocator to the available VM on the basis of the least cost factor. Therefore, the VM's updated status is received by the resource allocator to allocate the task for execution. Fig 1 demonstrates the resource allocation in a cloud model.

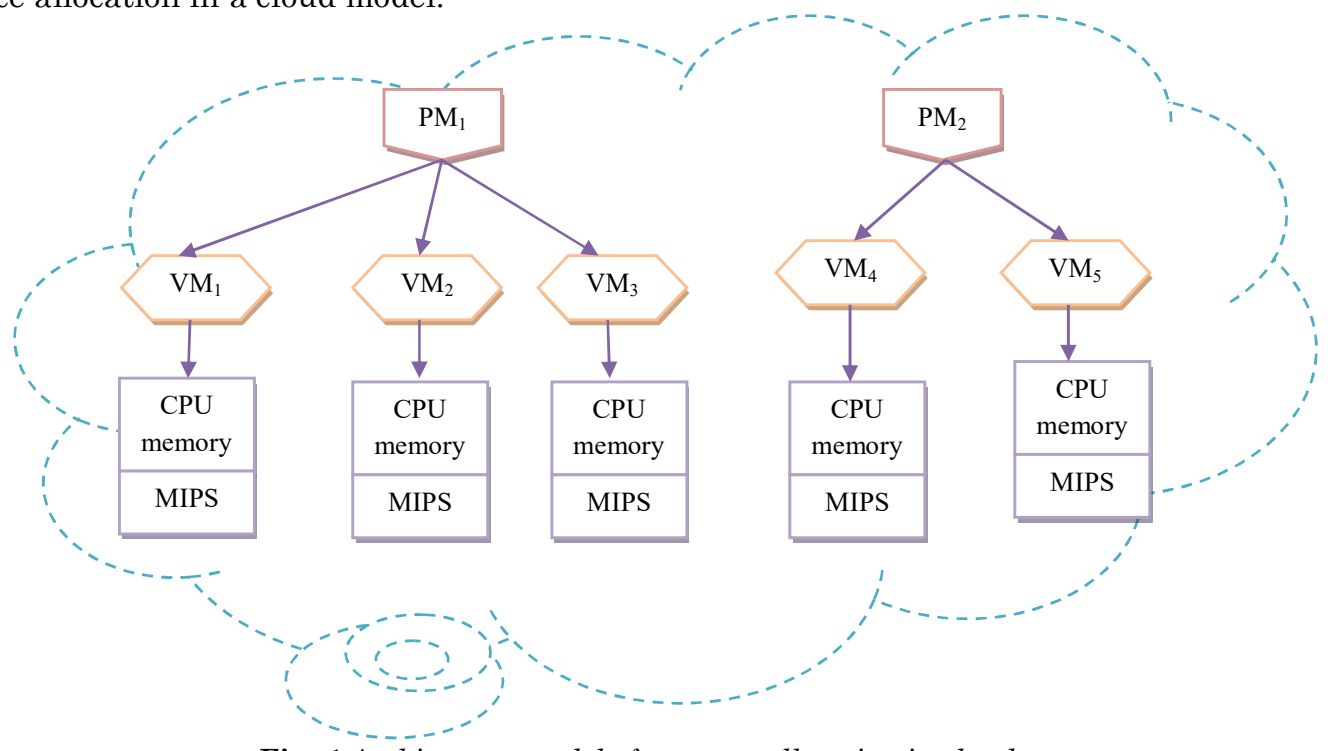

Fig. 1 Architecture model of resource allocation in cloud

\section{Resource Allocation in Cloud using Proposed Model}

In cloud provisioning services, resource allocation is considered a confronting task because of the service demands and reconfiguration needs. To attain effectual resource allocation, a novel computing model is developed in the cloud environment. In the cloud computing model, a resource allocation strategy is attained by the developed GWO-CSA model on the basis of the task chosen for the execution is performed. For each task, the execution is done using the developed model to effectually allocate the resources. On the basis of the proposed optimization technique, the resource allocation is done, which maximizes the cloud model efficiency. In the cloud environment, the resources are detached on enormous surfaces; therefore the allocation of resources is highly effectual. The VM is monitored and controlled by the PM so that every VM posses its individual CPU memory with MIPS speed.

The diverse cloud providers are composed of the resource allocation model which is connected to the VM, external and private. Presume $2 \mathrm{PM}$ indicated as, $\mathrm{P}_{1}$ and $\mathrm{P}_{2}$, and $5 \mathrm{VM}$ as $\mathrm{V}_{1}, \mathrm{~V}_{2}, \mathrm{~V}_{3}, \mathrm{~V}_{4}$, and $\mathrm{V}_{5}$, correspondingly. By exploiting the VM, the user assigned the task which is executed that is indicated as $\mathrm{V}=\left\{\mathrm{V}_{1}, \mathrm{~V}_{2}, \mathrm{~V}_{3}, \mathrm{~V}_{4}, \mathrm{~V}_{5}\right\}$. A denotes the applications which are established from the user. $s$ indicates each application possesses diverse tasks.

\subsection{Task flow}

Consider the task $s$ which is allocated to the VM on the basis of the availability of the resources. Here, 3 tasks are represented as $\left\{\mathrm{s}_{1}, \mathrm{~s}_{2}, \mathrm{~s}_{3}\right\}$ and start time of $\left\{\mathrm{S}_{1}, \mathrm{~S}_{2}, \mathrm{~S}_{3}\right\}$ the deadline for these tasks are indicated as $\left\{D_{1}, D_{2}, D_{3}\right\}$ and the runtime is $\left\{R_{1}, R_{2}, R_{3}\right\}$, correspondingly. Table 1 signifies the task flow for 3 diverse tasks besides their deadline, start time, and run time. To carry out the computation process the tasks are allocated to the VM. 
Table 1 Task flow

\begin{tabular}{cccc}
\hline Task & $\mathrm{s}_{1}$ & $\mathrm{~s}_{2}$ & $\mathrm{~s}_{3}$ \\
\hline Runtime & $\mathrm{R}_{1}$ & $\mathrm{R}_{2}$ & $\mathrm{R}_{3}$ \\
Deadline & $\mathrm{D}_{1}$ & $\mathrm{D}_{2}$ & $\mathrm{D}_{3}$ \\
Start time & $\mathrm{S}_{1}$ & $\mathrm{~S}_{2}$ & $\mathrm{~S}_{3}$ \\
\hline
\end{tabular}

For processing, while the cloud accepts the application, to the VM the tasks $\mathrm{s}_{1}, \mathrm{~s}_{2}$, as well as $\mathrm{s}_{3}$ are allocated by the proposed model. Nevertheless, on basis of the minimum cost of VM, the allocation is performed. To the VM, important factors are considered when tasks are allocated the parameters are deadline, cost as well as runtime. Both the private and external clouds are comprised of the cloud framework. In private clouds, tasks are mainly allocated to VM as the resource which does not request any extra cost. In the cloud, the task which posses' least cost is allocated to VMwhile tasks enter to evolution, the developed optimization technique examines the deadline and runtime of the task.

To efficiently carry out the resource allocation, the task with the least cost is allocated to VM. Consider 3 diverse applications as $\mathrm{T}_{1}, \mathrm{~T}_{2}$, and $\mathrm{T}_{3}$ and each of these applications possesses diverse tasks to execute. Table 2 summarizes the several tasks besides their start time as well as deadline. The deadline and runtime exploited to execute the tasks for each task $s$, at each application Tare evidently stated. The start time of the whole task $s$ is stated as 'one'. Here, tasks $s_{1}, s_{2}$, and $s_{3}$ are stated in the application $\mathrm{T}_{1}$, the task $\mathrm{s}_{4}$, and $\mathrm{s}_{5}$ are stated in the application $\mathrm{T}_{2}$, and the tasks $\mathrm{s}_{6}$ and $\mathrm{s}_{7}$ are stated in the application $\mathrm{T}_{3}$, correspondingly.

Table 2 Task for different applications

\begin{tabular}{lccccccc}
\hline Application & \multicolumn{3}{c}{ Application $\left(\mathrm{T}_{1}\right)$} & \multicolumn{2}{c}{ Application $\left(\mathrm{T}_{2}\right)$} & \multicolumn{2}{c}{ Application $\left(\mathrm{T}_{3}\right)$} \\
\hline Tasks & $\mathrm{s}_{1}$ & $\mathrm{~s}_{2}$ & $\mathrm{~s}_{3}$ & $\mathrm{~s}_{4}$ & $\mathrm{~s}_{5}$ & $\mathrm{~s}_{6}$ & $\mathrm{~s}_{7}$ \\
Runtime & 2 & 2 & 3 & 2 & 2 & 4 & 4 \\
Deadline & 5 & 2 & 7 & 5 & 3 & 8 & 8 \\
Start time & 1 & 1 & 1 & 1 & 1 & 1 & 1 \\
\hline
\end{tabular}

Table 3 summarizes the resource allocation within their particular runtime and deadline to the VM. The tasks $s$ are allocated V depend upon the least cost related to the task to the VM, and the allocation of the task to the VM is done by the adopted technique. Nevertheless, within runtime and deadline, the tasks are to be allocated in VM. Consider three diverse VM as $V_{1}, V_{2}$, and $V_{3}$, and presume $V_{1}$ encompasses the least cost subsequently $V_{2}$ and $V_{3}$. Therefore, the tasks are initially allocated to $\mathrm{V}_{1}$ subsequently $\mathrm{V}_{2}$ and $\mathrm{V}_{3}$, correspondingly. To the $\mathrm{VM}$ on the basis of the aforesaid value, the tasks are allocated so that task with the least value is allocated initially.

Table 3 Resource allocation within their runtime and deadline to the VM

\begin{tabular}{cccc}
\hline $\begin{array}{c}\text { Virtual machine/ } \\
\text { Time slots }\end{array}$ & $\mathrm{V}_{1}$ & $\mathrm{~V}_{2}$ & $\mathrm{~V}_{3}$ \\
\hline $\mathrm{Z}_{1}$ & $\mathrm{~s}_{2}$ & $\mathrm{~s}_{5}$ & $\mathrm{~s}_{7}$ \\
$\mathrm{Z}_{2}$ & $\mathrm{~s}_{2}$ & $\mathrm{~s}_{5}$ & $\mathrm{~s}_{7}$ \\
$\mathrm{Z}_{3}$ & $\mathrm{~s}_{1}$ & $\mathrm{~s}_{4}$ & $\mathrm{~s}_{7}$ \\
$\mathrm{Z}_{4}$ & $\mathrm{~s}_{1}$ & $\mathrm{~s}_{4}$ & $\mathrm{~s}_{7}$ \\
$\mathrm{Z}_{5}$ & $\mathrm{~s}_{3}$ & $\mathrm{~s}_{6}$ & - \\
$\mathrm{Z}_{6}$ & $\mathrm{~s}_{3}$ & $\mathrm{~s}_{6}$ & - \\
$\mathrm{Z}_{7}$ & $\mathrm{~s}_{3}$ & $\mathrm{~s}_{6}$ & - \\
$\mathrm{Z}_{8}$ & - & $\mathrm{s}_{6}$ & - \\
\hline
\end{tabular}

\subsection{For resource allocation in the cloud using the proposed model}

In $\mathrm{VM}$, the resource allocation is carried out, on the basis of the evaluation of the parameters like memory, CPU, MIPS, and skewness. On the basis of the minimum cost, the tasks are allocated using the developed optimization algorithm in VM. The developed GWO-CSA method is the combination of both 
the GWO [8] and CSA [9]. The resource allocation process is performed on the basis of the developed model with respect to the fitness value with the parameters. Fig 2 indicates the proposed model architecture for resource allocation in the cloud.

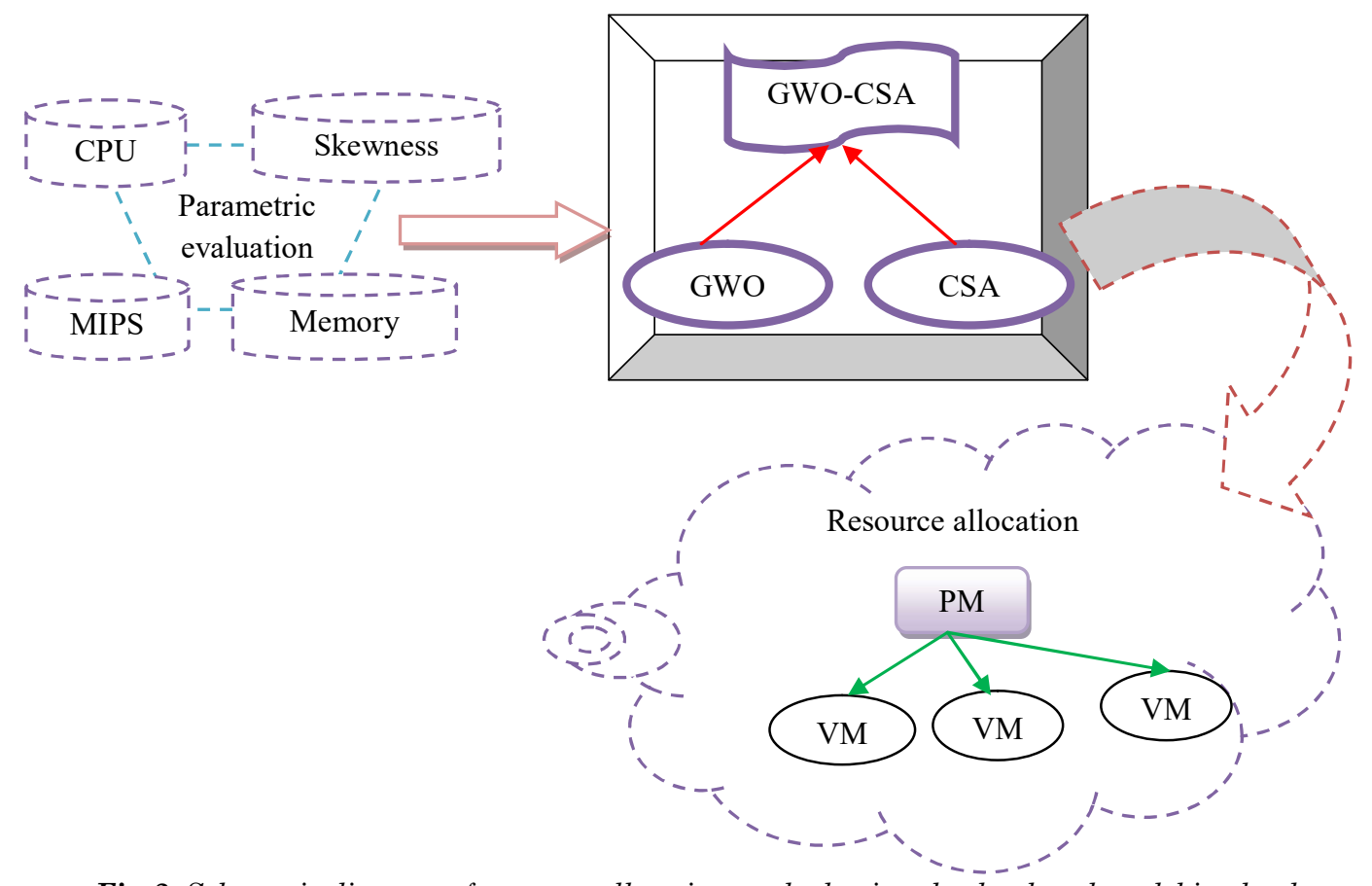

Fig 2. Schematic diagram of resource allocation method using the developed model in cloud

\subsection{Fitness evaluation}

To retrieve the optimal solution the fitness function is calculated. Here, the optimal solution is considered as a fitness function with a minimum value. Nevertheless, to calculate the fitness value the function is exploited and it is stated as

$$
\mathrm{f}=\sum_{\mathrm{m}=1}^{\mathrm{q}} \mathrm{A}_{\mathrm{m}}+\sum_{\mathrm{n}=1}^{\mathrm{p}}\left(\mathrm{F}_{\mathrm{n}}+\left(1-\mathrm{B}_{\mathrm{n}}\right)+\mathrm{G}_{\mathrm{n}}\right)
$$

In eq. (1), $F_{n}$ signifies the cost of $n^{\text {th }} V M, A_{m}$ signifies the runtime of $m^{\text {th }}$ task, $B_{n}$ signifies the resource utilization of $n^{\text {th }} V M, q$ signifies the total number of task, $G_{n}$ signifies the skewness, and, the term $B_{n}$ and $G_{n}$ is expressed as,

$$
\begin{aligned}
& \mathrm{B}_{\mathrm{n}}=\frac{\mathrm{L}_{\mathrm{n}}^{\mathrm{t}} \times \mathrm{Q}_{\mathrm{n}}^{\mathrm{v}} \times \mathrm{U}_{\mathrm{n}}^{\mathrm{v}}}{\mathrm{L}_{\mathrm{n}}^{\mathrm{t}} \times \mathrm{Q}_{\mathrm{n}}^{\mathrm{t}} \times \mathrm{U}_{\mathrm{n}}^{\mathrm{t}}} \times \frac{\mathrm{W}_{\mathrm{u}}}{\mathrm{W}_{\mathrm{x}}} \\
& \mathrm{G}_{\mathrm{n}}=\left(\frac{\mathrm{B}_{\mathrm{n}}}{\mathrm{B}}-1\right)^{2}
\end{aligned}
$$

$\mathrm{L}_{\mathrm{n}}^{\mathrm{U}}$ signifies the CPU used by $\mathrm{n}^{\text {th }} \mathrm{VM}, \mathrm{Q}_{\mathrm{n}}^{\mathrm{v}}$ signifies the memory used by $\mathrm{n}^{\text {th }} \mathrm{VM}, \mathrm{U}_{\mathrm{n}}^{\mathrm{v}}$ signifies the MIPS used by $n^{\text {th }}$ VM, $\mathrm{L}_{\mathrm{n}}^{\mathrm{t}}$ signifies the total CPU present in $\mathrm{n}^{\text {th }} \mathrm{VM}, \mathrm{Q}_{\mathrm{n}}^{\mathrm{t}}$ signifies memory present in $n^{\text {th }}$ VM, $U_{n}^{t}$ signifies MIPS present in $n^{\text {th }}$ VM, and $B$ signifies average resource consumption. $W_{u}$ signifies time slot consumption, and $\mathrm{W}_{\mathrm{x}}$ signifies utmost total slot.

\section{Proposed GWO-CSA MODEL}

Grey wolf optimizer (GWO)] is modeled as a cooperative method on the basis of the hunting behavior of grey wolves and the social leadership among them in nature [8].

Crow search algorithm (CSA) is modeled by Alireza Askarzadeh [9] on the basis of the nature intelligent of crows. a) Crows are found together as a flock. b) Crows can memorize the positions of their hiding places. c) Crows recognize victim's hiding place by pursuing each other. d) By a probability each crow protects food stores. 
The conventional GWO [13] and CSA [9] show some better performances on few unimodal benchmark function issues. Nevertheless, this algorithm deals in terms of highly complicated multimodal functions, ensnaring in local optima, and early convergence might be happened. Additionally, easy techniques' performance might degrade while dealing with large-scale dimensions. To surmount these disadvantages and enhance the exploring ability, a novel hybrid approach on the basis of the GWO and CSA is developed to resolve complex life issues and large-scale dimensions. The developed technique is named GWO-CSA. In the developed model, all grey wolves movements, and $\alpha$ grey wolf, are enhanced on the basis of the CSA to improve the diversity of the solutions, effectually. Additionally, a DFLS [10] on the basis of the information of optimal solution is developed to set up minute perturbation in the neighborhood of optimal until now result and then process the solution quality. Initially, on the basis of the opposition searching a learning scheme is introduced to protect crow's diversity. Next, to accelerate the future of optimal solutions, an iterative level hybridization through CSA is developed. Finally, to achieve top- solutions quality in each generation, a DFLS is modeled as a neighborhood searching scheme.

By taking into consideration of the awareness probability the crow is updated in CSA. While crow $j$ does aware that one more crow $i$ is pursuing it, subsequently crow $i$ will update its location arbitrarily. This might be deficient in the solution diversity and might be depreciated with the instantaneous convergence rate. Hence, rather than updating arbitrarily, a scheme on the basis of the opposition learning is modeled to protect crow's diversity as well as maximize exploration ability. The updating scheme is as stated below.

$$
x_{i, \text { iter }+1}=\left\{\begin{array}{ll}
x_{i, \text { iterq }}+r_{i} \cdot f_{i, \text { iter }} \cdot \\
\cdot\left(m_{j, \text { iter }}-x_{i, \text { iter }}\right) \\
q \cdot(u b+l b)-x_{i, \text { iter }}, & \text { if otherwise }
\end{array} \text { if } r_{j} \geq A P_{j, \text { iter }}\right.
$$

In eq. (4), $\mathrm{q}$ indicates a random number in $[0,1]$ and $\mathrm{lb}$ and $\mathrm{ub}$ denotes restrictions of the search space.

To execute both techniques in series iteratively this phase aspires to improve the performance of the optimization. Here, to obtain the gifted areas GWO is exploited as explore tool and CSA is subsequently permitted to use these areas to discover superior solutions. By exploiting the $\alpha, \beta, \delta$, and $\Delta$ wolves, the updating procedures of the candidate location are stated as below.

The 3 optimal crows indicated as $\Delta_{\text {crowl }}, \Delta_{\text {crow2 }}$ as well as $\Delta_{\text {crow3 }}$ are attained by exploiting fitness function subsequently they are analyzed with those generated via GWO $\left(\Delta_{\alpha}, \Delta_{\beta}, \Delta_{\delta}\right)$, to obtain the survival as follows:

$$
\left\{\begin{array}{l}
\Delta_{\alpha}=\arg \min \left\{\mathrm{f}\left(\Delta_{\alpha}\right), \mathrm{f}\left(\Delta_{\text {crowl }}\right)\right\} \\
\Delta_{\beta}=\arg \min \left\{\mathrm{f}\left(\Delta_{\beta}\right), \mathrm{f}\left(\Delta_{\text {crow2 }}\right)\right\} \\
\Delta_{\delta}=\arg \min \left\{\mathrm{f}\left(\Delta_{\delta}\right), \mathrm{f}\left(\Delta_{\text {crow } 3}\right)\right\}
\end{array}\right.
$$

\section{Experimental Procedure}

In this section, performance evaluation was performed by the developed model regarding the evaluation metrics. Here, the developed model was analyzed by the performance measures namely, memory utilization, resource utilization, CPU utilization, and skewness, correspondingly. Moreover, the proposed method is compared with the conventional models such as Particle Swarm Optimization (PSO), Grey Wolf Optimization (GWO) and Genetic Algorithm (GA).

Figs 3, 4, and 5 summarize the performance analysis on the basis of the optimal performance attained by the comparative techniques. In Fig 3, the proposed method is 35\% better than the PSO, $28 \%$ better than the GWO and 20\% better than the GA for CPU resource utilization. In Fig 4, the proposed method is $43 \%$ better than the PSO, $42 \%$ better than the GWO and $40 \%$ better than the GA for Memory utilization. In Fig 5, the proposed method is $45 \%$ better than the PSO, $10 \%$ better than the GWO and $9 \%$ better than the GA for Resource utilization. 


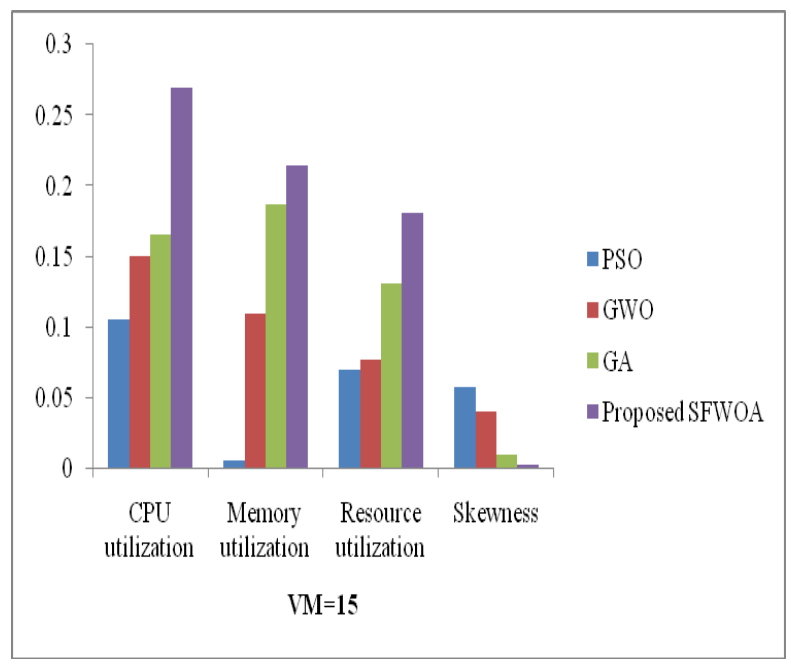

Fig 3 Performance analysis of proposed model for $V M=15$

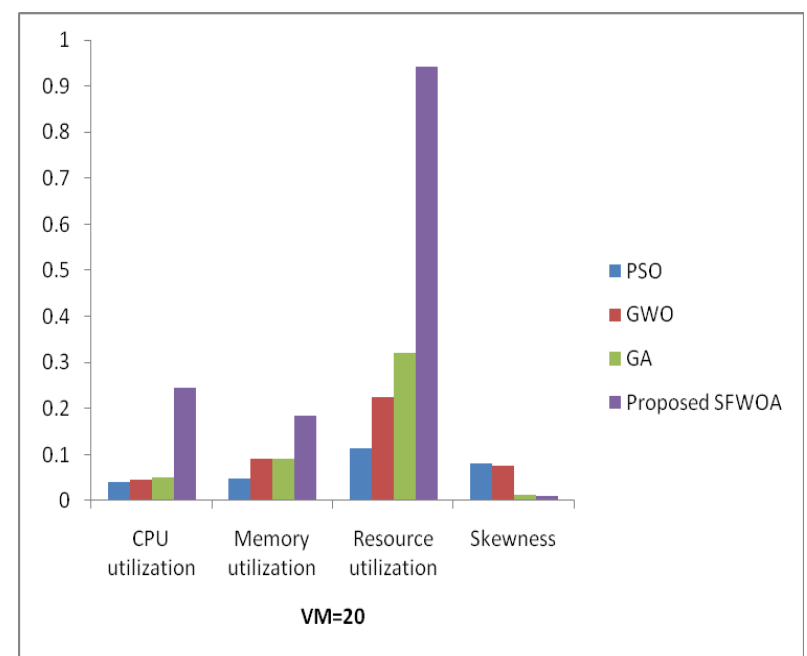

Fig 4 Performance analysis of a proposed model for $V M=20$

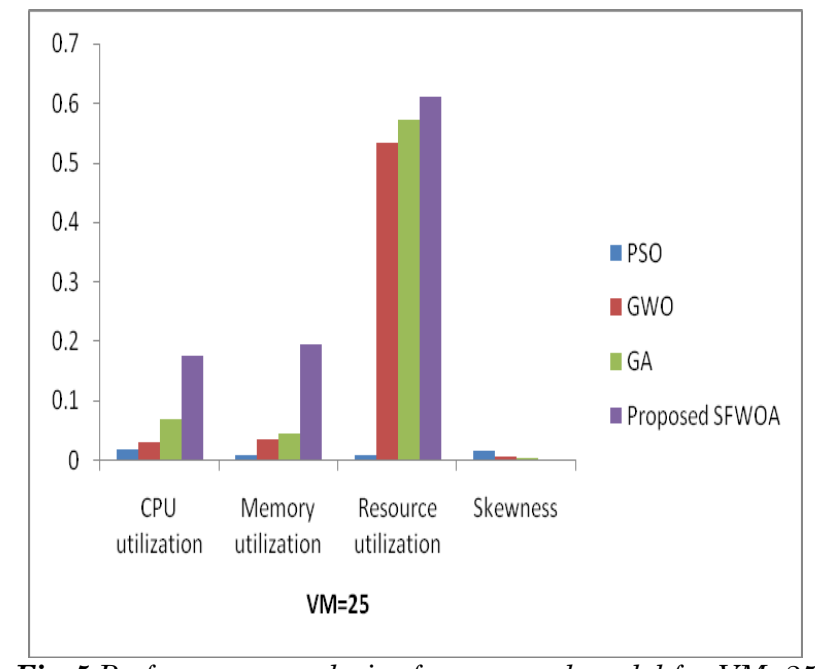

Fig 5 Performance analysis of a proposed model for $V M=25$

\section{Conclusion}

In the cloud framework, resource allocation creates the computing paradigm in an effective way. Moreover, a resource allocation model in an optimization-driven way was effectually developed for resource allocation in the cloud without performance degradation in a system. Using the proposed model, 
to the virtual machine the task was efficiently allocated. Here, the adopted model was the integration of both the GWO and CSA models. In the cloud model, the maximum resource utilization and the minimal cost were exploited by the fitness function. On the basis of the parameters such as virtual machine cost, runtime, and skewness, the fitness function was derived to define the allocation scheme. The proposed method was exploited for the task with the least cost was allocated to the virtual machine by taking into consideration of the deadline and runtime. Here, the optimal solution was considered as the minimum value in the fitness function. Finally, the proposed model attained overall better performances compared with the conventional models.

\section{References}

[1] Ritu SinghalArchana Singhal,"A feedback-based combinatorial fair economical double auction resource allocation model for cloud computing", Future Generation Computer Systems,8 October 2020.

[2] Jixian ZhangXutao YangWeidong Li,"An online auction mechanism for time-varying multidimensional resource allocation in clouds", Future Generation Computer Systems,25 April 2020.

[3] Kalka DubeyS. C. Sharma,"An extended intelligent water drop approach for efficient VM allocation in secure cloud computing framework", Journal of King Saud University - Computer and Information SciencesAvailable online, 10 November 2020.

[4] Jing Li,"Resource optimization scheduling and allocation for hierarchical distributed cloud service system in smart city", Future Generation Computer Systems2 January 2020.

[5] Junyan HuKenli LiKeqin Li,"Coalition formation for deadline-constrained resource procurement in cloud computing", Journal of Parallel and Distributed Computing,28 October 2020.

[6] Christina Terese JosephK. Chandrasekaran,"IntMA: Dynamic Interaction-aware resource allocation for containerized microservices in cloud environments", Journal of Systems Architecture1 May 2020.

[7] Durao, F., Carvalho, J.F.S., Fonseka, A. and Garcia, V.C., "A systematic review on cloud computing", The Journal of Supercomputing, vol. 68, no. 3, pp.1321-1346, 2014.

[8] V. Yadav, G. Parmar and R. Bhatt, "Application of GWO with Different Performance Indices for BH System," 2019 4th International Conference on Information Systems and Computer Networks (ISCON), Mathura, India, pp. $742-745,2019$.

[9] S. Shirke and R. Udayakumar, "Evaluation of Crow Search Algorithm (CSA) for Optimization in Discrete Applications," 2019 3rd International Conference on Trends in Electronics and Informatics (ICOEI), Tirunelveli, India, pp. 584-589, 2019.

[10] L. A. Zadeh, "Fuzzy sets," Inf. Control, vol. 8, no. 3, pp. 338-353, June. 1965.

[11] Michael Mahesh K,"Workflow Scheduling using Improved Moth Swarm Optimization Algorithm in Cloud Computing",Multimedia Research, vol. 3, no. 3, July 2020.

[12] VhatkarKapilNetaji,Bhole G P,"Optimal Container Resource Allocation Using Hybrid SA-MFO Algorithm in Cloud Architecture", Multimedia Research, vol. 3, no. 1, January 2020

[13] V. Vinolin,"Breast Cancer Detection by Optimal Classification using GWO Algorithm", Multimedia Research, vol. 2, no. 2, April 2019. 